\title{
A Highly Selective Staurosporine Derivative Designed by a New Selectivity Filter
}

\author{
Ibrahim M. El-Deeb, ${ }^{\dagger, \dagger}$ Su Jin Jung, ${ }^{\S}$ Byung Sun Park, ${ }^{\dagger}$ Young Jun Yoo, ${ }^{\dagger}$ Kihang Choi, ${ }^{\S}$ \\ Young Mok Yang, ${ }^{\#}$ Sang Woo Lee, ${ }^{\uparrow}$ In Tae Kim, ${ }^{\natural}$ Dong Keun Han, ${ }^{\dagger, *}$ and So Ha Lee ${ }^{\dagger, *}$ \\ ${ }^{\dagger}$ Center for Biomaterials, Korea Institute of Science and Technology, P.O. Box 131, Cheongryang, Seoul 130-650, Korea \\ EE-mail:LSH6211@kist.re.kr,dkh@kist.re.kr \\ ${ }^{\star}$ Department of Biomolecular Science, University of Science and Technology, Daejeon 305-333, Korea \\ ${ }^{\S}$ Department of Chemistry, Korea University, Seoul 136-701, Korea \\ ${ }^{\#}$ Department of Pathology, School of Medicine, Konkuk University, Chungju City 380-701, Korea \\ "Department of Chemistry, Kwangwoon University, Seoul 139-701, Korea \\ Received January 18, 2011, Accepted March 7, 2011
}

\begin{abstract}
KIST301135 was semi-synthetically prepared by the reaction of Staurosporine with triphosgene in anhydrous dichloromethane. The structure of KIST301135 was confirmed by ${ }^{1} \mathrm{H}$ NMR, ${ }^{13} \mathrm{C}$ NMR, and high resolution mass spectrum. KIST301135 was initially tested in a single dose duplicate mode at a concentration of $20 \mathrm{nM}$ over a panel of 53 kinases against Staurosporine as a positive control. KIST301135 has showed inhibitions above $75 \%$ in only 2 kinases (FLT3 and JAK3 kinases) of the 53 tested kinases, while Staurosporine has showed inhibitions above $80 \%$ in about $62 \%$ of the tested kinases. KIST301135 was retested at a 5-dose testing mode over the 9 kinases inhibited by percentages over 20 at the single dose testing in order to determine its IC $_{50}$ values. KIST301135 has shown much improved kinase inhibitory selectivity relative to Staurosporine in its potency at JAK3 kinase and CAMK2b kinase.
\end{abstract}

Key Words : Staurosporine derivative, Selectivity filter, Kinase selectivity, Cancer

\section{Introduction}

Kinases are important targets in molecular cancer therapy, and the inhibition of oncogenic kinases involved in the initiation and/or progression of different types of cancers has become an attractive and promising approach for treatment of such cancers. However, the evolutionary relatedness and structural conservation of these proteins are often responsible of unexpected cross-reactivities, ${ }^{1,2}$ yielding uncertain or even life-threatening side effects upon inhibition with non-selective kinase inhibitors. ${ }^{3-5}$ Accordingly, the therapeutic use of non-selective inhibitors may be potentially hazardous unless a rational strategy to control their specificity is adopted. Such control may be achieved if selectivity filters in the target protein can be identified. These selectivity filters could be any structural features that are unique to the target, and are liable for discrimination and distinction by a uniquely designed inhibitor drug. Thus, much of the cross reactivity may be removed by redesign guided by the identification of structural features that promote crossreactivity and selectivity filters that enable target discrimination. $^{6-9}$

Staurosporine is a natural competitive inhibitor that binds to the ATP pocket of almost all kinases in the active conformation (the activation loop is fully extended and exposed to solvent). ${ }^{10-12}$ Staurosporine has a unique skeleton, that is larger and more rigid than that of ATP, with a solvent accessible surface area of $360 \AA^{2}$ (vs $323 \AA^{2}$ for ATP). ${ }^{12}$ The broad range of Staurosporine activity over the majority of kinases is due to the numerous contacts it makes with conserved polar and hydrophobic groups in protein kinases. It is the most cross-reactive kinase inhibitor known to date, ${ }^{1,2}$ where it was found to bind tightly (with an $\mathrm{IC}_{50}$ below 3.0 $\mu \mathrm{M})$ to $\sim 90 \%$ of the 290 human kinases screened through phage-display ATP-competitive assays. ${ }^{2}$ Such high levels of cross reactivity make it impossible to use Staurosporine as a therapeutic agent. Thus, despite its inhibitory potency, Staurosporine is solely regarded as a research compound. A number of synthetic and semi-synthetic Staurosporine analogues were developed and evaluated, aiming to improve their kinase selectivity profiles, and enabling their clinical use as anticancer drugs. Examples for these analogues are UCN-01, UCN-02, PKC-412, LY-333531 and AFN-941, which are all chemically modified at different parts of Staurosporine skeleton (Fig. 1). ${ }^{13,14}$ Some of these derivatives have showed improvements in their kinase inhibitory selectivity and are currently at different stages of clinical trials. However, the improvements in selectivity in these new inhibitors were associated, in most of the cases, with a big decrease in potency at the selectively inhibited proteins.

A selectivity filter, which was used to improve Staurosporine selectivity, has been recently reported by Crespo et al. and has utilized what so called "the packing defects of soluble proteins". ${ }^{15}$ In this selectivity filter, the unique solvent-exposed intramolecular backbone hydrogen bonds (called dehydrons), which are not conserved across kinases families, are targeted through the insertion of non-polar groups into the inhibitor that wrap these dehydrons (protect them from water attack), and hence impart more selectivity and favorability for the interaction with this inhibitor. This 


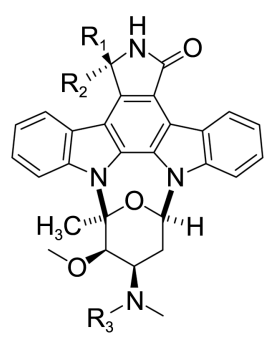

Staurosporine: $\mathrm{R}_{1}, \mathrm{R}_{2}, \mathrm{R}_{3}=\mathrm{H}$ UCN-01: $\mathrm{R}_{1}=\mathrm{OH}, \mathrm{R}_{2}, \mathrm{R}_{3}=\mathrm{H}$

UCN-02: $\mathrm{R}_{1}, \mathrm{R}_{3}=\mathrm{H}, \mathrm{R}_{2}=\mathrm{OH}$

PKC-412: $\mathrm{R}_{1}, \mathrm{R}_{2}=\mathrm{H}, \mathrm{R}_{3}=$ benzoyl

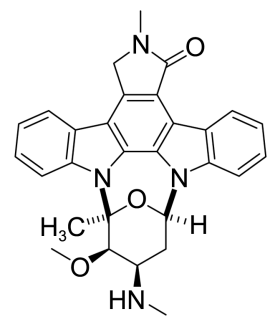

6-Methyl staurosporine

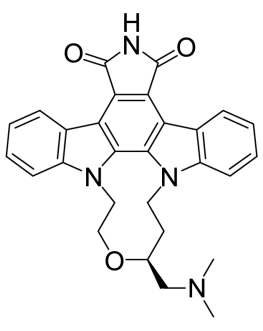

LY-333531

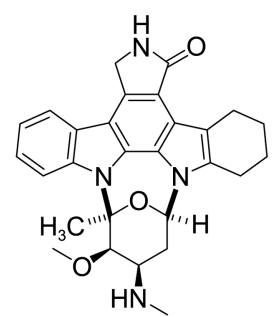

AFN-941
Figure 1. Structures of Staurosporine, its analogues, and the dimer inhibitor KIST301135.

approach was applied to Staurosporine, where a small wrapping methyl group was introduced to its lactam NH (6Methyl Staurosporine; Fig. 1). This newly introduced methyl group has improved the selectivity profile of the derivative by wrapping a unique dehydron in a small group of kinases, and at the same time, destroying a key hydrogen bonding interaction in a large number of kinases. This methyl wrapping has restricted the compound inhibitory impact to the kinases that share this dehydron at the aligned position. However, this improvement in selectivity was on expense of potency, which was greatly reduced at the selectively inhibited kinases, since a highly essential hydrogen bonding interaction was lost by the introduction of the wrapping methyl group.

Herein, we report a simple and effective redesign for the most non-selective kinase inhibitor known as Staurosporine, where a Staurosporine dimer KIST301135 was synthesized, by linking two Staurosporine molecules through their aminomethyl side chains using a carbonyl bridge (Fig. 1). The resulted dimer, KIST301135, has expressed much improved kinase inhibitory selectivity relative to the parent compound, Staurosporine, without much decrease in its potency at the fewer number of selectively inhibited kinases.

\section{Results and Discussion}

The proposed explanation for the great improvement in the selectivity of the new Staurosporine-dimer, KIST301135, without significant decrease in its potency is based on a new type of selectivity filters (size exclusion filter) that utilizes the relative variations in binding pockets' geometry in different kinases as its filtration tool. According to this theory, the enlargement of the inhibitor size (doubling its size by dimer

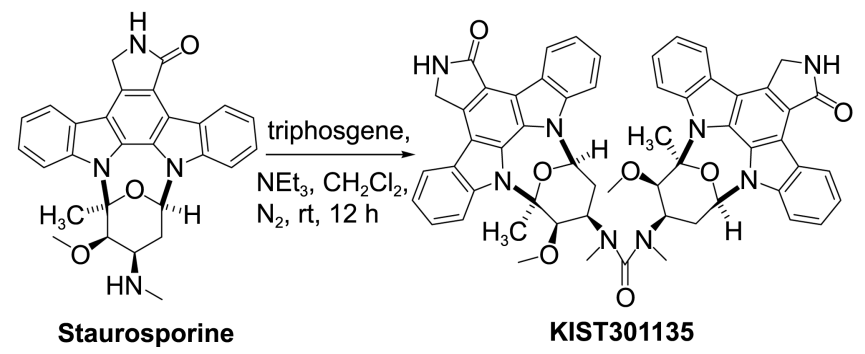

Scheme 1. Synthetic route of KIST301135.

formation) would result in a bigger molecule that is still capable of accommodation within the geometry of certain kinases (those with the suitable large binding pocket) without clashing with any surface residues, while at the same time is too large to interact with the relatively smaller kinases' pockets. This theory is quite different from the concept of "bifunctional molecules" where two protein binding moieties are joined together via a linker of suitable length so as to dimer two protein monomers in one time. ${ }^{16}$ In the theory, the linker combining the two molecules must be very short (preferably one atom in length) in order to avoid bifunctional interaction, contrasting for the "bifunctional molecules" in which the linker should be long enough to allow the binding of the two functionalities to two protein molecules in the same time.

The target compound KIST301135 was semi-synthetically prepared starting from Staurosporine, by allowing Staurosporine to react with triphosgene in anhydrous dichloromethane, and in the presence of triethylamine at room temperature for a period of $12 \mathrm{~h}$, to yield the target compound in $13 \%$ yield (Scheme 1). The structure of the target compound was confirmed by ${ }^{1} \mathrm{H}$ NMR, ${ }^{13} \mathrm{C}$ NMR and mass spectra. The dimerization through the side chain -NH- and not the lactam -NH- was confirmed by the ${ }^{1} \mathrm{H}$ NMR of the compound in DMSO- $d_{6}$, where the highly deshielded singlet peak corresponding to the lactam -NH- was observed at a $\delta$ value of $8.69 \mathrm{ppm}$. The peak corresponding to the methyl protons of the side chain $-\mathrm{OCH}_{3}$ could not be observed in the ${ }^{1} \mathrm{H}$ NMR spectra recorded in DMSO- $d_{6}$ and $\mathrm{CD}_{3} \mathrm{OD}$. In both of these two solvents, the peak was hidden behind the water peak (in the range of 3.30-3.40 ppm), however, the presence of this methyl was confirmed by the correct molecular ion peak in mass spectrum corresponding to the assigned structure of KIST301135 with a molecular mass value of 959.06 . The presence of this methyl group was also confirmed by the ${ }^{13} \mathrm{C}$ NMR spectrum of KIST301135 recorded in DMSO- $d_{6}$, where the peak corresponding to this methyl was observed at a $\delta$ value of $30.02 \mathrm{ppm}$ as shown in the experimental section.

In order to investigate the kinase inhibitory profile of KIST301135 and compare its selectivity with that of Staurosporine, KIST301135 was initially tested in a single dose duplicate mode at a concentration of $20 \mathrm{nM}$ over a panel of 53 kinases against Staurosporine as a positive control. The screening was carried out at Reaction Biology Corporation using Hot Spot Technique. ${ }^{17}$ KIST301135 has showed much improved selectivity profile over the tested kinases relative 


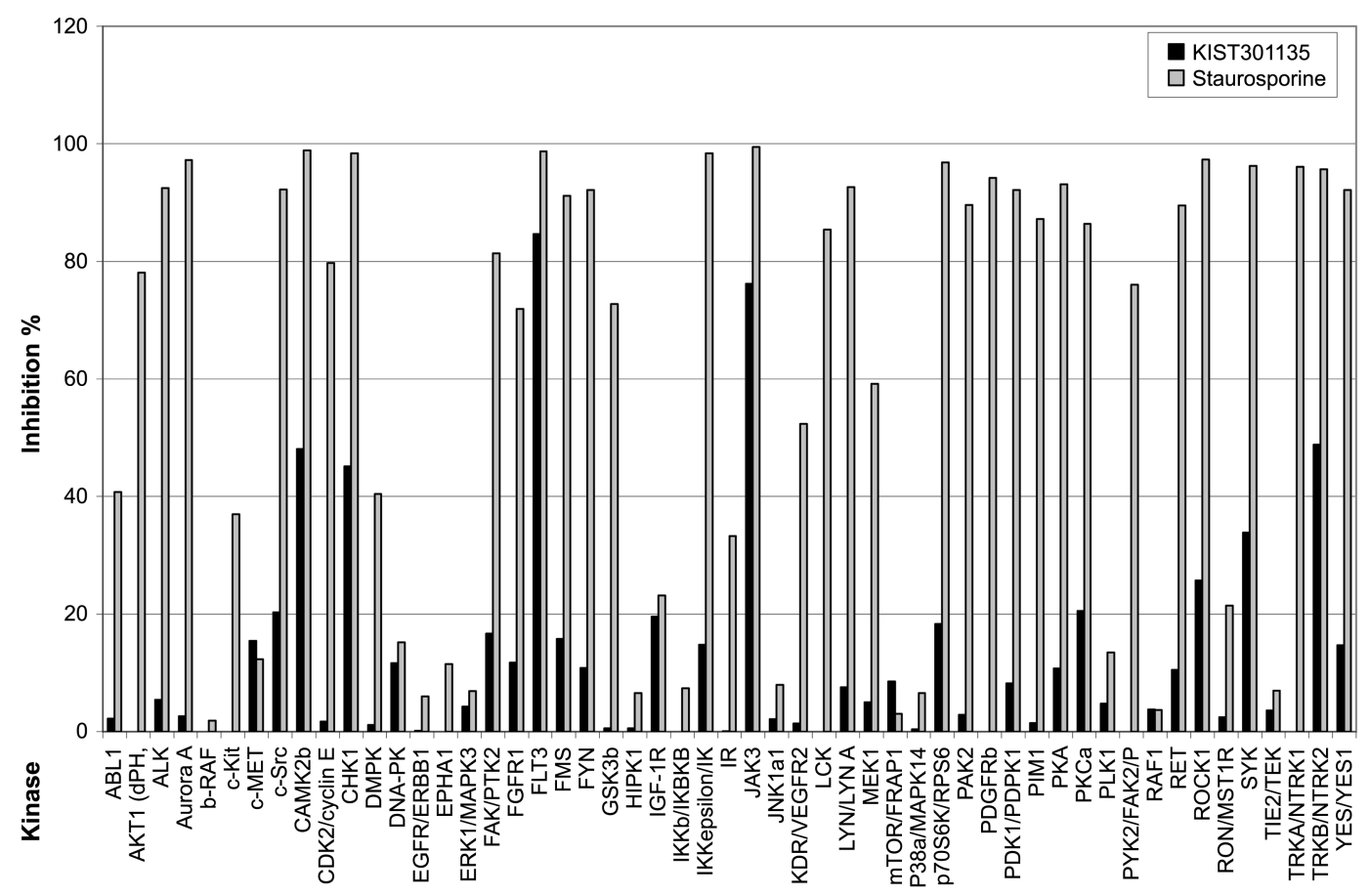

Figure 2. Inhibition percentages of KIST301135 and Staurosporine at a single dose concentration of $20 \mathrm{nM}$ over 53 kinases.

Table 1. $\mathrm{IC}_{50}$ values in $\mathrm{nM}$ of KIST301135 and Staurosporine over the 9 most sensitive kinases

\begin{tabular}{ccc}
\hline Kinase & KIST301135 $(\mathrm{nM})$ & Staurosporine $(\mathrm{nM})$ \\
\hline JAK3 & 0.26 & $<0.5$ \\
CAMK2b & 0.38 & $<0.5$ \\
FLT3 & 1.63 & $<0.5$ \\
CHK1 & 2.19 & $<0.5$ \\
TRKB & 2.50 & $<0.5$ \\
SYK & 16.21 & 0.57 \\
ROCK1 & 64.84 & $<0.5$ \\
$c$-Src & 534.60 & 0.72 \\
IGF-1R & 5589.00 & 29.79 \\
\hline
\end{tabular}

to Staurosporine (Fig. 2). At the test concentration, KIST301135 has showed inhibitions above $75 \%$ in only 2 kinases (FLT3 and JAK3 kinases) of the 53 tested kinases (3.7\% of the whole kinase panel), while the inhibition percentages were below $50 \%$ at all of the other kinases under test. On the other hand, Staurosporine has showed inhibitions above $80 \%$ in about $62 \%$ of the tested kinases (33 of the 53 tested kinases) when tested at the same single dose concentration $(20 \mathrm{nM})$.

In order to make the comparison more clear, KIST301135 was retested at a 5-dose testing mode over the 9 kinases inhibited by percentages over 20 at the single dose testing for its IC $_{50}$ values over these 9 kinases (Table 1). KIST301135 has showed comparable potencies to that of Staurosporine at JAK3 kinase (Janus kinase 3, whose mutations are associated with different autosomal SCID "severe combined immunodeficiency disease") ${ }^{18}$ and CAMK2b kinase (whose activity is elevated in cardiac arrhythmias and other heart diseases). ${ }^{19}$ The potencies were slightly lower than that of Staurosporine, but still in a single digit nM scale over FLT3 kinase (mutated in acute myeloid leukemia "AML"), ${ }^{20} \mathrm{CHK} 1$ kinase (a cellcycle kinase whose inhibition promotes apoptosis) ${ }^{21}$ and TrkB (which mediates resistance to chemotherapy in neuroblastomas). ${ }^{22}$ The potencies at SYK and ROCK1 kinases were lower than that of Staurosporine, although still below $100 \mathrm{nM}$, while the potency was weak at c-Src and much weaker at IGF-1R, indicating the presence of these two kinases beyond the selectivity range of the new compound.

According to our proposed theory of size exclusion, the improved selectivity of KIST301135 over the tested kinases is thought to be caused by the excessive bulkiness of the molecule which precludes it from entering the kinase domains of a large number of the kinases that are normally and strongly inhibited with Staurosporine. This results finally in a geometrical selectivity for KIST301135 to a limited number of kinases whose binding pocket can accommodate this bulky compound. It's not anticipated however that the whole molecule would be accommodated inside the binding pockets of these kinases, it's rather proposed that the compound would occupy a similar binding pose to that of Staurosporine through one half of the molecule (one of the two Staurosporine moieties), while the second half of the compound would be extruding outside the binding pocket.

This extruding half is so critical in determining the kinase inhibitory potency of KIST301135 relative to that of the parent Staurosporine. It is assumed that in very few cases, the extruding molecule would be able to establish extra bindings with the external residues at the outer boundaries of the kinase pocket, leading to more potentiated inhibitory effect relative to that of Staurosporine, while in the majority of cases, this extruding part would clash with some surface residues at the enzyme, leading to weakened and unfavo- 


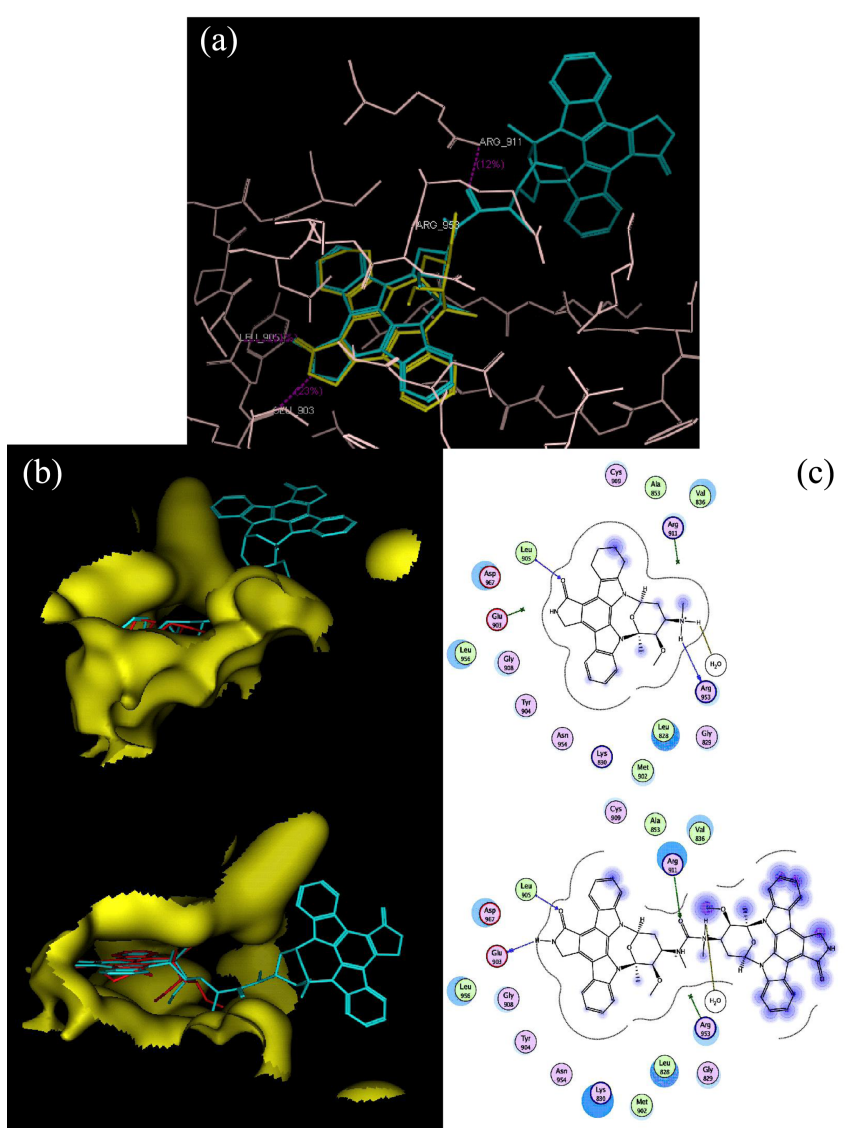

Figure 3. Molecular modeling data. (a) Overlay of the structures of KIST301135 (cyan) and AFN941 (yellow) in the binding pocket of JAK3 kinase. (b) 3D binding of KIST301135 (cyan) and AFN941 (red) to the binding pocket of JAK3 kinase showing one half of KIST301135 extruding outside the pocket. Side view (upper panel), top view (lower panel). (c) 2D binding interactions of AFN941 (upper panel) and KIST301135 (lower panel) to JAK3 kinase.

rable binding of the compound to these kinases, and that would be the key point which determines the compound selectivity. In a third case, the extruding part would neither impart any significant binding nor clashes with kinase surfaces, producing inhibitory effect that is more or less similar to that of Staurosporine.

A molecular modeling study was applied using MOE. 2008.10 software, in order to have a better molecular insight at these speculations about the new inhibitor KIST301135 and its binding interactions, and identify the possible binding mode of KIST301135 to the kinase domain of JAK3 kinase. JAK3 kinase was selected since it is the most strongly inhibited kinase with KIST301135, and also because of the availability of a co-crystal structure of JAK3 kinase with the tertahydro-Staurosporine analogue AFN941 ( $\mathrm{Pdb}=$ 1YVJ). Accordingly, KIST301135 was docked into the receptor atoms of JAK3 kinase and its most stable binding mode was compared with that of the co-crystallized Staurosporine analogue, AFN941. It was found that KIST301135 occupies a highly identical binding pose to that of AFN941, and a perfect overlay was observed between the two struc- tures (Fig. 3(a)).

As assumed in the previous discussions, it could be observed that one of the two halves of the compound binds typically to the same residues occupied by AFN941 (and similarly Staurosporine), while the other half extrudes outside the binding pocket (Fig. 3). The compound was fit well into the binding pocket of the enzyme forming typical hydrogen bonding (HB) and hydrophobic interactions to those of AFN941. The compound has established two key HB interactions with leucine 905 and glutamate 903 through its $\mathrm{HB}$ acceptor lactam carbonyl and its $\mathrm{HB}$ donor amide $\mathrm{NH}$, respectively (Fig. 3(c)). Furthermore, the bridging carbonyl linking two halves of the molecule was found to share in extra binding with the surface residues of the binding pocket, through binding to arginine 911, which could result finally in a stronger and more potentiated binding, and consequently inhibition, of this kinase, relative to Staurosporine (Fig. 3(c)).

In addition to the anticipated therapeutic benefits from using KIST301135 in treatment of diseases associated with abnormalities in the selectively inhibited kinases such as JAK3 and FLT3, the high affinity of the compound to these kinases along with its strong fluorescence characters qualify the compound to be used also as a molecular imaging agent for such kinases. ${ }^{23}$ The UV-visible and fluorescence spectroscopic characters of KIST301135 have been recorded in methanol and compared with those of Staurosporine. It was found that KIST301135 absorbs UV radiation with a $\lambda_{\max }$ value of $293 \mathrm{~nm}$, which is typical to that of Staurosporine. The irradiation of KIST301135 and Staurosporine solutions in methanol at $5 \mathrm{nM}$ concentrations by a single beam UV light at the $\lambda_{\max }$ of their absorption spectra $(293 \mathrm{~nm})$ produced highly similar luminescence spectra showing two luminescence maxima at $373 \mathrm{~nm}$ and $395 \mathrm{~nm}$ in KIST301135 and at $379 \mathrm{~nm}$ and $399 \mathrm{~nm}$ in Staurosporine. The intensity of luminescence produced by KIST301135 is much higher than that of Staurosporine, and accordingly the bioluminescence of KIST301135 within living cells could be utilized to measure the activities of JAK3 and FLT3 kinases.

In summary, we have successfully redesigned the most non-selective kinase inhibitor known "Staurosporine" producing a highly selective and an equipotent new inhibitor "KIST301135" using a new selectivity filter which utilizes the differences in binding pockets' geometry in different kinases as the filtration tool. Our new inhibitor "KIST301135" has showed much improved kinase inhibitory selectivity relative to Staurosporine, without a significant decrease in potency. The improved selectivity of KIST301135 along with its preserved high potency at kinases such as JAK3 and FLT3 qualify it to be a potential drug candidate for the treatment of diseases associated with impairments in such kinases. The new size exclusion approach presented in this study provides a new simple and efficient method for the redesign of a large number of potent but non-selective kinase inhibitors already available, in order to qualify them to be employed as more selective inhibitors in different treatments. The theory could be also extended to "synergistic 
therapies" by joining two different inhibitors known to potentiate the effect of each other, where a double benefit would be expected in such case, by improving the selectivity of both inhibitors in addition to increasing their activities.

\section{Experimental}

General Methods. ${ }^{1} \mathrm{H}$ NMR $(300 \mathrm{MHz})$ and ${ }^{13} \mathrm{C}$ NMR (75 MHz) were recorded on a Bruker Avance 300 spectrometer with TMS as an internal standard. Mass spectra were recorded on a JMS-700 (JEOL, Japan), using Fast Atom Bombardment (FAB) for ionization with FAB energy of 6 $\mathrm{KeV}$, emission of $5 \mathrm{~mA}$, and acceleration voltage of $10 \mathrm{kv}$. Melting points were taken on a Thomas- Hoover capillary melting apparatus and were uncorrected. UV-visible spectra and luminescence spectra were recorded on S-2130 spectroscopy and RF-5301-PC luminescent spectroscopy, respectively. Column chromatography was performed on Merck silica gel 60 (230-400 mesh). TLC was carried out using glass sheets precoated with silica gel 60 F254 prepared by E. Merck. All the commercially available reagents were obtained from Aldrich and Tokyo Kasei Chemicals and generally used without further purification.

1,3-Bis((9S,10R,11R,13R)-2,3,10,11,12,13-hexahydro-10methoxy-9-methyl-9,13-epoxy-1H,9H-diindolo[1,2,3-gh: 3',2',1'-Im]pyrrolo [3,4-j][1,7]benzodiazonin-1-one)-1,3dimethylurea (KIST301135). To a stirred solution of Staurosporine (100 mg, $0.21 \mathrm{mmole}$ ) and triethylamine (54 $\mathrm{mg}, 0.54 \mathrm{mmole})$ in anhydrous dichloromethane $(2 \mathrm{~mL})$ at $0{ }^{\circ} \mathrm{C}$ was added a solution of triphosgene $(38 \mathrm{mg}, 0.13$ $\mathrm{mmol})$ in anhydrous dichloromethane $(1 \mathrm{~mL})$ in a dropwise manner over a period of 10 minutes. The ice bath was removed, and stirring was maintained at room temperature for $12 \mathrm{~h}$. The solution was concentrated in vacuo, and the residue was purified by column chromatography (silica gel, ethyl acetate-hexane, 7:1, v/v) to yield pure KIST301135 as yellowish white powder. Yield (13.4 mg, 13\%), mp 296-298 ${ }^{\circ} \mathrm{C},{ }^{1} \mathrm{H}$ NMR $\left(\mathrm{CD}_{3} \mathrm{OD}\right): \delta 2.08\left(\mathrm{~s}, 6 \mathrm{H}, \mathrm{CH}_{3}\right), 2.25$ (dd, $J=$ 8.4, 9.0 Hz, 2H), $2.64\left(\mathrm{~s}, 6 \mathrm{H}, \mathrm{NCH}_{3}\right), 2.90(\mathrm{~m}, 2 \mathrm{H}), 3.31(\mathrm{~s}$, $\left.6 \mathrm{H}, \mathrm{OCH}_{3}\right), 4.38$ (br s, $\left.2 \mathrm{H}\right), 5.04$ (br s, $\left.4 \mathrm{H}\right), 5.26$ (d, $J=6.0$ $\mathrm{Hz}, 2 \mathrm{H}), 6.81(\mathrm{t}, J=5.4 \mathrm{~Hz}, 2 \mathrm{H}), 6.81(\mathrm{t}, J=5.4 \mathrm{~Hz}, 2 \mathrm{H})$, $7.30(\mathrm{t}, J=11.4 \mathrm{~Hz}, 2 \mathrm{H}), 7.38(\mathrm{t}, J=10.8 \mathrm{~Hz}, 2 \mathrm{H}), 7.47-7.52$ (m, 4H), $7.64(\mathrm{~d}, J=6.0 \mathrm{~Hz}, 2 \mathrm{H}), 7.99(\mathrm{~d}, J=5.4 \mathrm{~Hz}, 2 \mathrm{H})$, $8.10(\mathrm{~d}, J=6.3 \mathrm{~Hz}, 2 \mathrm{H}), 9.20(\mathrm{~d}, J=5.7 \mathrm{~Hz}, 2 \mathrm{H}),{ }^{1} \mathrm{H} \mathrm{NMR}$ (DMSO- $\left.d_{6}\right): \delta 1.93(\mathrm{~m}, 2 \mathrm{H}), 2.02\left(\mathrm{~s}, 6 \mathrm{H}, \mathrm{CH}_{3}\right), 2.58(\mathrm{~s}, 6 \mathrm{H}$, $\left.\mathrm{NCH}_{3}\right), 2.91(\mathrm{~m}, 2 \mathrm{H}), 3.37\left(\mathrm{~s}, 6 \mathrm{H}, \mathrm{OCH}_{3}\right), 4.33(\mathrm{~m}, 2 \mathrm{H})$, 4.98-5.06 (m, 4H), $5.29(\mathrm{~d}, J=8.6 \mathrm{~Hz}, 2 \mathrm{H}), 6.96(\mathrm{dd}, J=$ 3.6, $6.0 \mathrm{~Hz}, 2 \mathrm{H}), 7.30(\mathrm{t}, J=7.6 \mathrm{~Hz}, 2 \mathrm{H}), 7.38(\mathrm{t}, J=7.7 \mathrm{~Hz}$, 2H), $7.51(\mathrm{t}, J=8.1 \mathrm{~Hz}, 4 \mathrm{H}), 7.78(\mathrm{~d}, J=8.4 \mathrm{~Hz}, 2 \mathrm{H}), 8.04$ (t, $J=7.8 \mathrm{~Hz}, 4 \mathrm{H}), 8.69$ (s, 2H, NH), 9.22 (d, $J=7.8 \mathrm{~Hz}$, $2 \mathrm{H}),{ }^{13} \mathrm{C}$ NMR (DMSO- $\left.d_{6}\right): \delta 28.71,29.21,30.02,45.96$, 52.54, 75.87, 79.63, 92.98, 109.20, 115.89, 116.27, 117.11, $120.07,120.76,121.45,121.70122 .84,125.06,125.45$, $125.98,126.23,129.06,133.43,136.88,140.82,156.12$, 172.06, FAB HRMS $m / e$ calcd for $(\mathrm{M}+\mathrm{H}) \mathrm{C}_{57} \mathrm{H}_{51} \mathrm{~N}_{8} \mathrm{O}_{6}$ 959.3802, found 959.3881, UV $\lambda_{\max }=293 \mathrm{~nm}\left(\mathrm{CH}_{3} \mathrm{OH}\right) ; \mathrm{PL}$ $\lambda_{\max }=373,395 \mathrm{~nm}\left(\mathrm{CH}_{3} \mathrm{OH}\right)$.
Kinase Screening. Kinase assays were performed at Reaction Biology Corporation using the 'HotSpot' assay platform. Kinase assay protocol, reaction buffer: base reaction buffer, $20 \mathrm{mM}$ Hepes ( $\mathrm{pH}=7.5), 10 \mathrm{mM} \mathrm{MgCl}, 1 \mathrm{mM}$ EGTA, 0.02\% Brij35, $0.02 \mathrm{mg} / \mathrm{mL}$ BSA, $0.1 \mathrm{mM} \mathrm{Na} \mathrm{VO}_{4}, 2$ $\mathrm{mM}$ DTT, 1\% DMSO. Required cofactors were added individually (if needed) to each kinase reaction. Reaction Procedure: To a freshly prepared buffer solution was added any required cofactor for the enzymatic reaction, followed by the addition of the selected kinase at a concentration of 20 $\mu \mathrm{M}$. The contents were mixed gently, and then KIST301135 dissolved in DMSO was added to the reaction mixture in the appropriate concentration. 339-ATP (specific activity 500 $\mu \mathrm{Ci} / \mu \mathrm{L}$ ) was added to the mixture in order to initiate the reaction, and the mixture was incubated at room temperature for $2 \mathrm{~h}$. Initial screening over 53 kinases: KIST301135 was tested by single dose duplicate made at a concentration of 20 nM. Staurosporine was used as a control compound in a 5dose $\mathrm{IC}_{50}$ mode with 10 -fold serial dilutions starting at 20 $\mu \mathrm{M}$. Reaction was carried out at $10 \mu \mathrm{M}$ ATP concentration. $\mathrm{IC}_{50}$ testing: KIST301135 was tested in a 10 -dose $\mathrm{IC}_{50}$ mode with three-fold serial dilutions starting at $5 \mu \mathrm{M}$. Staurosporine was used as a control compound in a 10-dose $\mathrm{IC}_{50}$ mode with three-fold serial dilutions starting at $10 \mu \mathrm{M}$. Reaction was carried out at $10 \mu \mathrm{M}$ ATP concentration.

Molecular Modeling. Docking studies were performed using "Molecular Operating Environment (MOE) version 2008.10", Chemical Computing Group Inc., 1010 Sherbrooke Street West, Suite 910, Montreal, H3A 2R7, Canada. KIST301135 was built using the builder interface of the MOE program and subjected to energy minimization using the included Forcefield MMFF94x calculations. The X-ray crystallographic structure of JAK3 kinase complexed with AFN941 (Pdb ID: 1YVJ) was obtained from Protein Data Bank. ${ }^{24}$ The enzyme was prepared for docking studies as follows: (i) hydrogen atoms were added to the structure with their standard geometry. (ii) the whole protein structure containing the ligand inside was subjected to energy minimization to relax any abnormally strained parts of the structure, (iii) the ligand KIST301135 was docked into the binding site, setting the receptor to "receptor + solvent", the site to "pocket atoms", and the pharmacophore to "none". Placement method was set to default "Triangle Matcher", the first scoring function, "rescoring 1" was set to the default "London $\mathrm{dG}$ " and the retain dropdown was set to 10 . Refinement inside the binding site was allowed using forcefield calculations so as to allow energy minimization of the docking poses inside the binding pocket. The refinement scoring function "rescoring 2" was set to none and the retain dropdown was set to 10 . The final refined poses were ranked by the MM/GBVI binding free energy estimation, and the pose showing the lowest free energy (the most stable pose) was selected.

Acknowledgments. This research was supported by the Pioneer Research Center Program through the National Research Foundation of Korea funded by the Ministry of Education, Science and Technology (2010-0002170), the 
Fundamental R\&D Program for Core Technology of Materials funded by the Ministry of Knowledge Economy, Korea (K0006028), and a research grant of Kwangwon University (2010). We would like to express our gratitude and thanks to Dr. Sean W. Deacon and Dr. Haiching Ma from Reaction Biology Corporation for carrying out the kinase screening.

\section{References}

1. Fabian, M. A.; Biggs, W. A.; Treiber, D. K.; Atteridge, C. E.; Azimioara, M. D.; Benedetti, M. G.; Carter, T.; Ciceri, P.; Edeen, P. T.; Floyd, M.; Ford, J. M.; Galvin, M.; Gerlach, J. L.; Grotzfeld, R. M.; Herrgand, S.; Insko, D. E.; Insko, M. A.; Lai, A.; Lelias, J. M.; Mehta, S.; Milanov, Z. V.; Velasco, A. M.; Wodicka, L. M.; Patel, H. K.; Zirrankar, P. P.; Lockhart, D. A. Nat. Biotechnol. 2005, 23, 329.

2. Karaman, M. K.; Herrgard, S.; Treiber, D. K.; Gallant, P.; Atteridge, C. E.; Campbell, B. T.; Chan, K. W.; Ciceri, P.; Davis, M. I.; Edeen, P. T.; Faraoni, R.; Floyd, M.; Hunt, J. P.; Lockhart, D. J.; Milanov, Z. V.; Morrison, M. J.; Pallares, G.; Patel, H. K.; Pritchard, S.; Wodicka, L. M.; Zarrinkar, P. P. Nat. Biotechnol. 2008, 26, 127.

3. Druker, B. J. Oncologist 2004, 9, 357.

4. Kerkela, R.; Grazette, L.; Yacobi, R.; Iliescu, C.; Patten, R.; Beahm, C.; Walters, B.; Shevtsov, S.; Pesant, S.; Clubb, F. J.; Rosenzweig, A.; Salomon, R. N.; Van Etten, R. A.; Alroy, J.; Durand, J.; Force, T. Nat. Med. 2006, 12, 908.

5. Force, T.; Krause, D. S.; Van Etten, R. A. Nat. Rev. Cancer 2007, 7,332 .

6. Fernandez, A.; Sanguino, A.; Peng, Z.; Ozturk, E.; Chen, J.; Crespo, A.; Wulf, S.; Shavrin, A.; Qin, C.; Ma, J.; Trent, J.; Lin, Y.; Han, H.; Mangala, L. S.; Bankson, J. A.; Gelovani, J.; Samarel, A.; Bornmann, W.; Sood, A. K.; Lopez-Berestein, G. J. Clin.
Invest. 2007, 117, 4044.

7. Demetri, G. J. Clin. Invest. 2007, 117, 3650

8. Crunkhorn, S. Nat. Rev. Drug Discov. 2008, 7, 120.

9. Crespo, A.; Fernandez, A. Drug Discov. Today 2007, 12, 917.

10. Fernandez, A.; Maddipati, S. J. Med. Chem. 2006, 49, 3092.

11. Zhao, B.; Bower, M. J.; McDevitt, P. J.; Zhao, H.; Davis, S. T.; Johanson, K. O.; Green, S. M.; Concha, N. O.; Zhoud, B. S. J. Biol. Chem. 2002, 277, 46609.

12. Atwell, S.; Adams, J. M.; Badger, J.; Buchanan, M. D.; Feil, I. K.; Froning, K. J.; Gao, X.; Hendle, J.; Keegan, K.; Leon, B. C.; Muller-Dieckmann, H. J.; Nienaber, V. L.; Noland, B. W.; Post, K.; Rajashankar, K. R.; Ramos, A.; Russell, M.; Burley, S. K.; Buchanan, S. G. J. Biol. Chem. 2004, 279, 55827.

13. Gescher, A. Gen. Pharmacol. 1998, 31, 721.

14. Boggon, T. J.; Li, Y.; Manley, P. W.; Eck, M. J. Blood 2005, 106, 996.

15. Crespo, A.; Zhang, X.; Fernández, A. J. Med. Chem. 2008, 51, 4890.

16. Corson, T. W.; Aberle, N. C.; Crews, M. ACS Chem. Biol. 2008, 3, 677.

17. Reaction Biology Corporation web site, www.reactionbiology.com.

18. Notarangelo, L. D.; Mella, P.; Jones, A.; de Saint Basile, G.; Savoldi, G.; Cranston, T.; Vihinen, M.; Schumacher, R. F. Hum. Mutat. 2001, 18, 255 .

19. Anderson, M. E. Pharmacol. Ther. 2005, 106, 39.

20. Schmidt-Arras, D.; Schwäble, J.; Böhmer, F. D.; Serve, H. Curr. Pharm. Des. 2004, 10, 1867.

21. Damia, G.; Broggini, M. Cell Cycle 2004, 3, 46.

22. Eggert, A.; Ikegaki, N.; Liu, X. G.; Brodeur, G. M. Klin. Padiatr. 2000, 212, 200.

23. Zhang, L.; Lee, K. C.; Bhojani, M. S.; Khan, A. P.; Shilman, A.; Holland, E. C.; Ross, B. D.; Rehemtulla, A. Nat. Med. 2007, 13, 1114.

24. www.rcsb.org/pdb. 